Vol. 14, No. 52, July 2019, 1201-1215

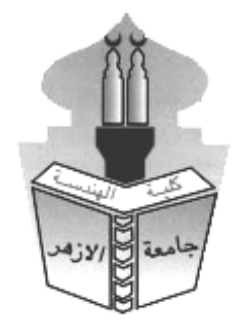

\title{
THE ECONOMIES OF THE BIO-CLIMATIC DESIGN APPLIED STUDY FOR TYCOONS BUILDING PROJECT (IN NEW CAIRO)
}

\author{
Algendy Shaker Abdelghany Algendyand Anessa Hassan Mohammed Abou Elwafa \\ Department of Architecture - Faculty of Engineering - Al-Azhar University - Egypt \\ a.shaker@azhar.edu.eg \\ nogahassan49@gmail.com
}

\begin{abstract}
Architecture experienced many subsequent mutations in the latest decades to develope buildings, target of the research is to find a conclusion of standards and strategies that must be provided to achieve an economic bioclimatic building with consideration of the surrounding environmental conditions to achieve thermal comfort for gap users. the study also includes strategies to achieve a bioclimatic design and an applied study for a tycoons building project in altajamue alkhamis. this applied study amis to test what has been reached . methodology of the applied study includes the setting of goals, the determination of the study field and the used tools in the form of analysis tables and standards.it ends with the implementation of the study and drawing conclusions.
\end{abstract}

Skywards : Bio-climatic Architecture

$$
\begin{aligned}
& \text { اقتصاديات التصميم البيومناخى التئي }
\end{aligned}
$$

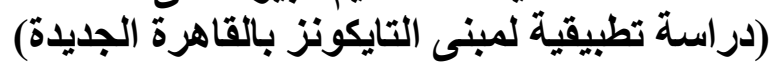

$$
\begin{aligned}
& \text { الجندى شاكر عبد الغنى الجندى و أنيسة حسن محمد أبو الوفا }
\end{aligned}
$$

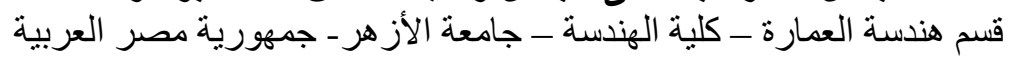

شهدت العمارة عدة طفرات متلاحقة فى العقود الأخيرة أدت إلى تطوير المبانى حيث يهدف البحث إلى استنتاج المعايير

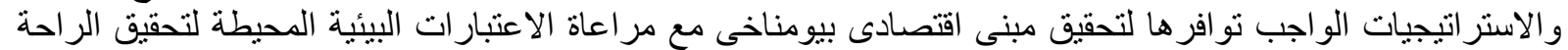

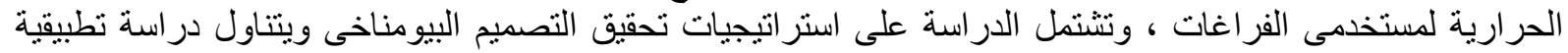

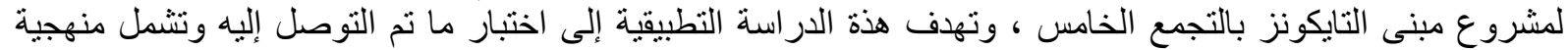

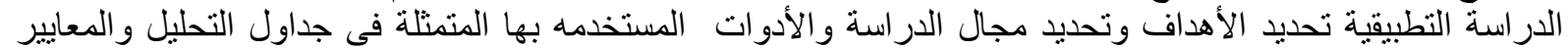

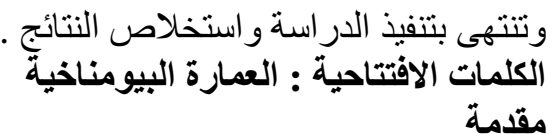

مع الطلب الدائم لمستخدمى الفراغ على تحقيق الراحة الحرارية داخل الفراغات المعمارية توجه المستخدم إلى استخدام

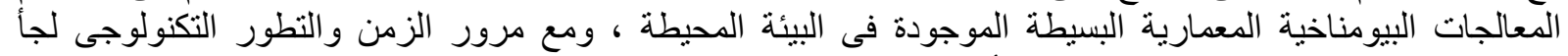

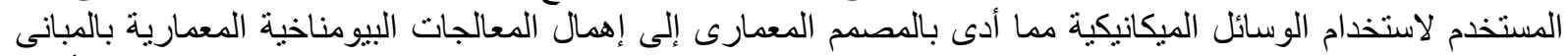

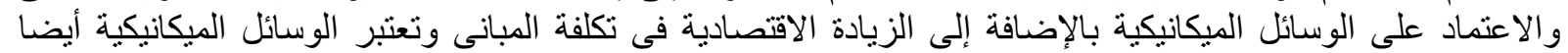

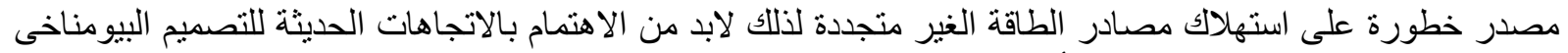

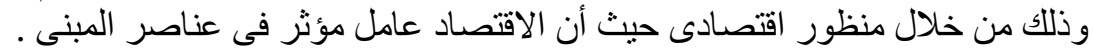

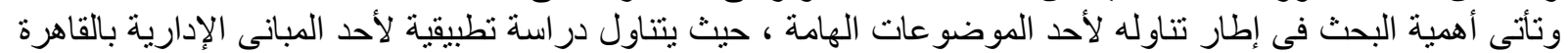

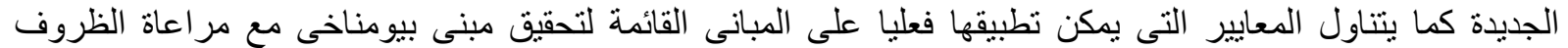




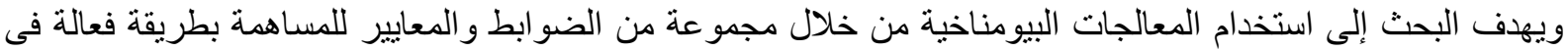
تحقيق عمارة اقتصادية ذات بيئة بيومناخية ، ويتم تطبيق هذة المعالجات و المعايير على مبنى التايكونز بالقاهرة الجديدة

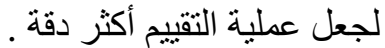
ويتبع البحث المنهج الاستقر ائى والتحليلى والتطبيقى ، للتعرف على دور وأهمية المبانى البيومناخية فى تحقيق الراحة

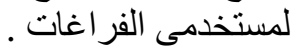

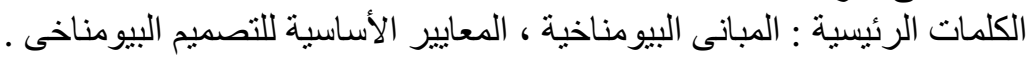

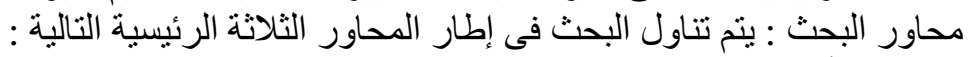

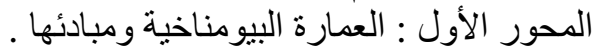

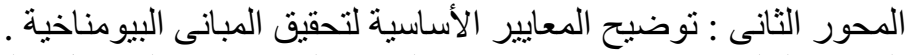

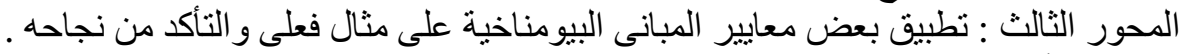

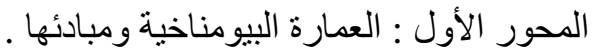

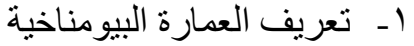

يوجد تعريفات عديدة للعمارة البيومناخية ، لكن من القر اءات المتعددة يمكن تعريف العمارة البيومناخية على أنها العملية

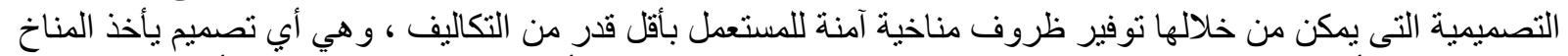

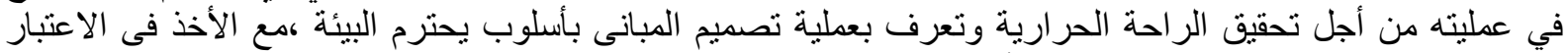

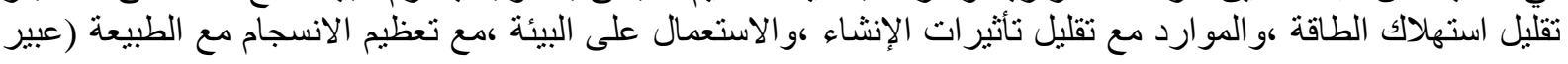

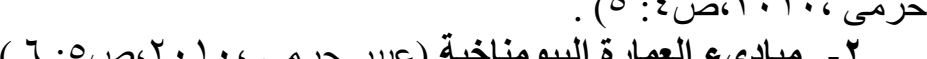

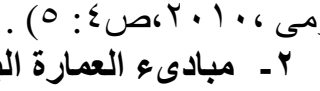

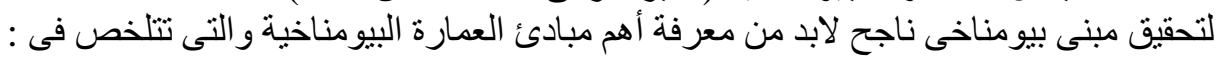

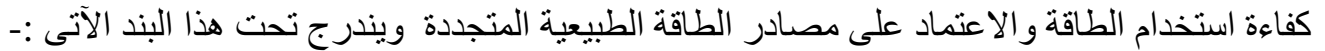

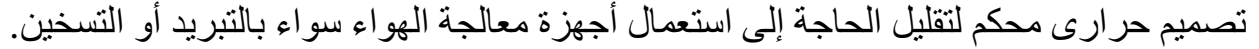

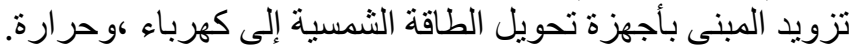
البعد البيئى و الذى يلعب دورا كبير ا فى عملية التصميم الأخضر من حيث :

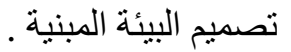
تاثير البناء على البيئة الطبيعية . البئة

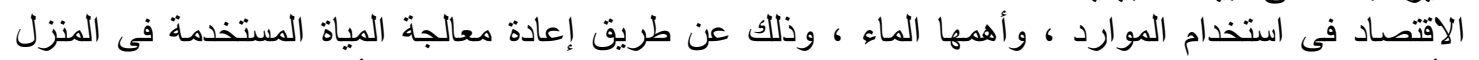

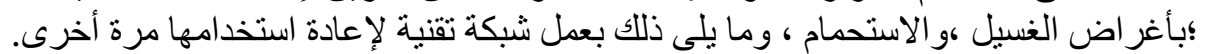

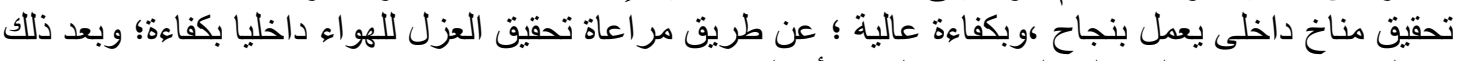

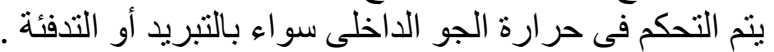

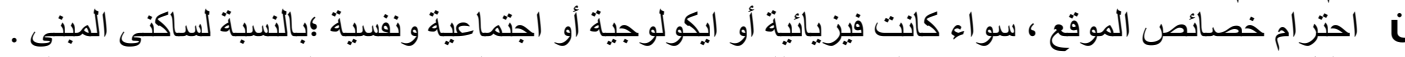

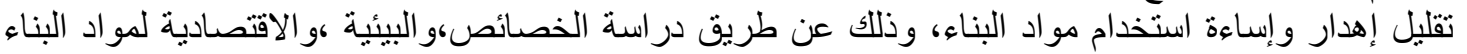

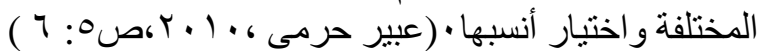

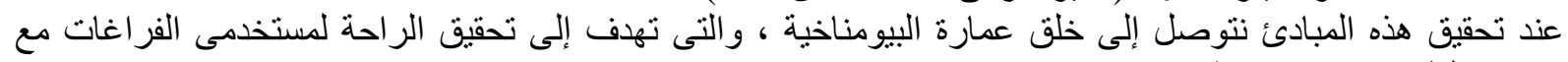

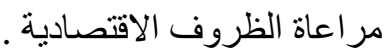
المحور الثانى : توضيح الأوضانة المعايير الأساسية لتحقيق المبانى البيومناخية

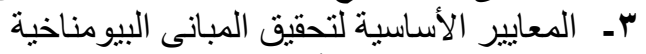
هناك مجمو عة من المعايير الأساسية لتحقيق مبنى بيومناخى ذاتئية كفاءة التصادية يمكن تلخيصها فى الجدول الآتى : 
جدول (1 ـ 1) يوضح المعايير الأساسية لتصميم المبنى البيومناخى (المصدر: الباحث)

\begin{tabular}{|c|c|c|}
\hline \multicolumn{3}{|l|}{ المعايير الأساسية لتصميم المبنى البيومناخى } \\
\hline 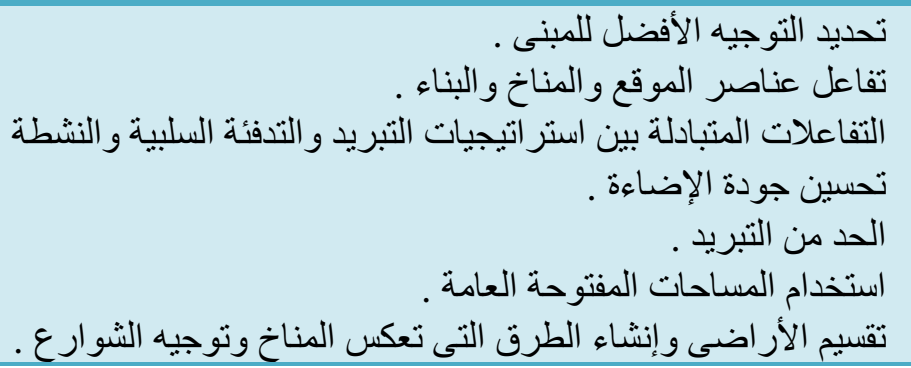 & • & البيو مناخى على مستوى التصميم \\
\hline 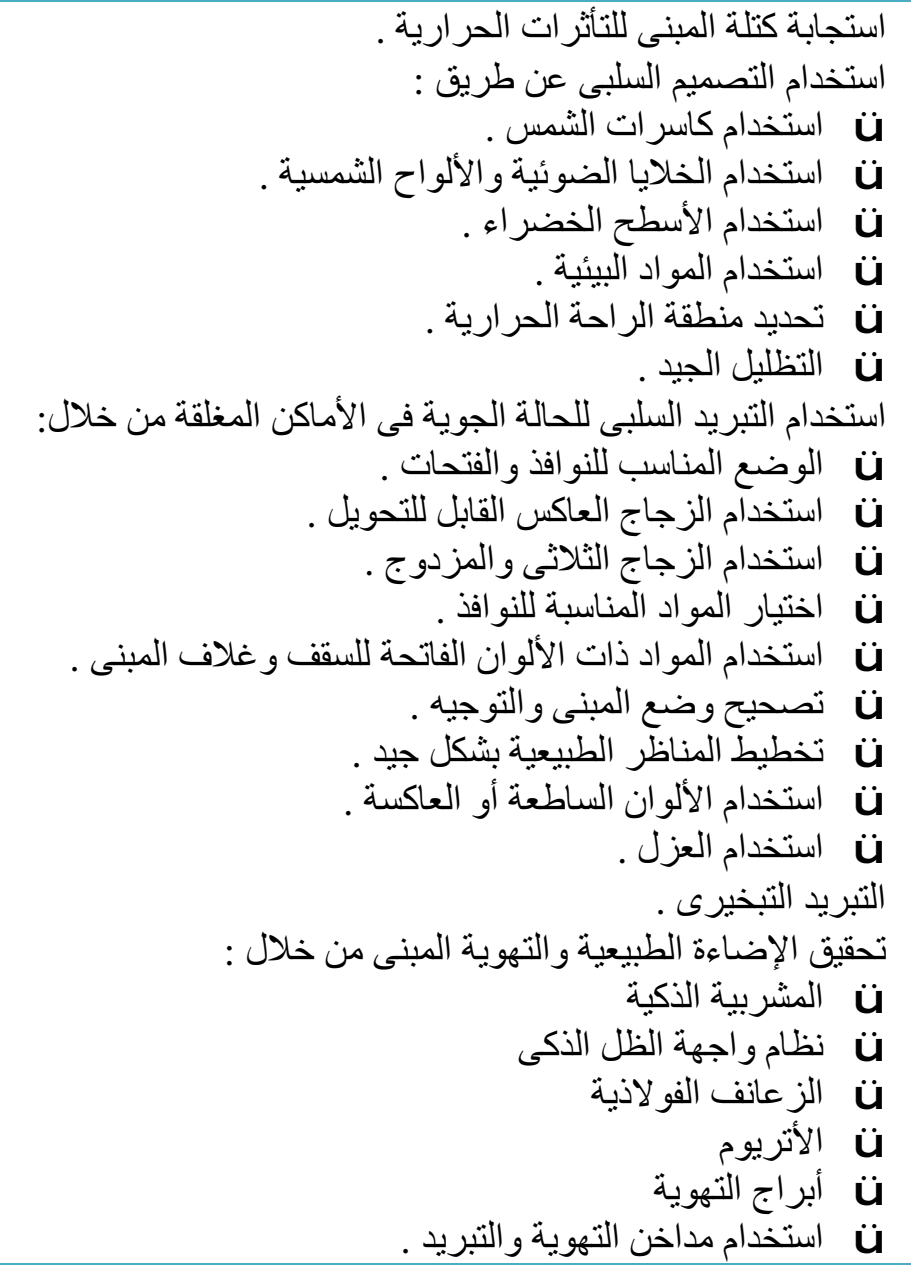 & • & 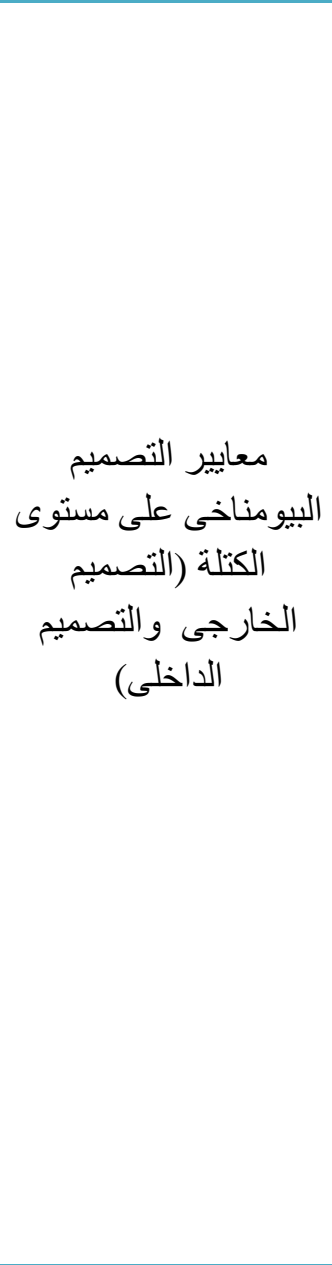 \\
\hline 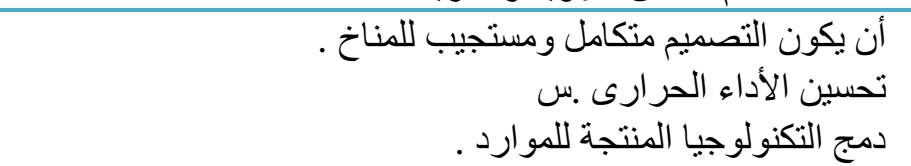 & • & البيو مناخى على مسلتوى التصميم \\
\hline
\end{tabular}

المحور الثالث : تطبيق بعض معايير المبانى البيومناخية على مبنى التايكونز بالقاهرة الجديدة .

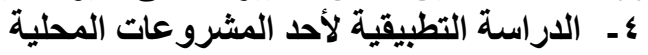

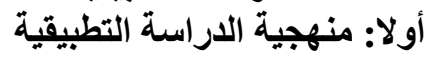

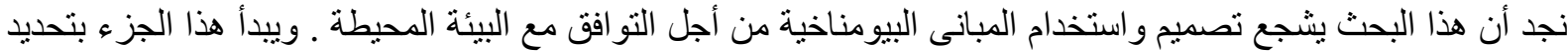

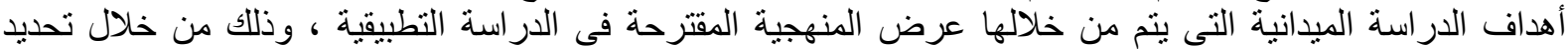

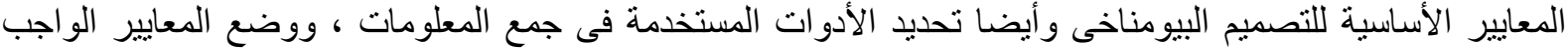

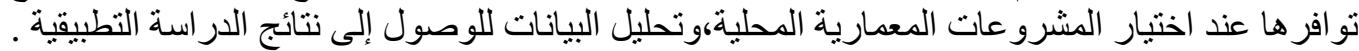


تهدف الدر اسة الميدانية إلى تحقيق الآتى إلى

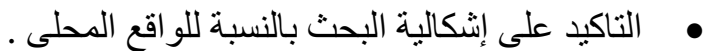

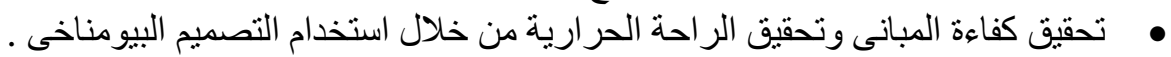

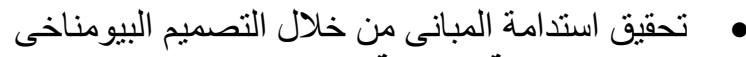
ثالثا: معايير اختيار عينة الاراسة التئة

تم اختيار عينة الذراسة من خينة خلال الأمنلة التى تحقق المعايير الأساسية النالية

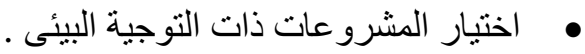

ا اختبار مبنى إدارى يتكون من عدد من المكاتب وذللك لأن المبانى الإدارية من أكثر أنواع المبانى استهلاكا للطاقة

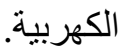

رابعا: عينة الدراسة استة

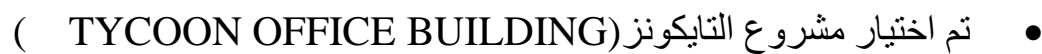
سوف نقوم بعمل در اسة تطبيقية على مبنى التايكونز بتطبيق هذة المعايير على المبنى لتحقيق التصميم البيومناخى مع

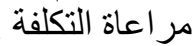

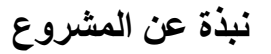
يقع المشروع فئ مدينة القاهرة الجديدة التى تقع بالجانب الثرقى من القاهرة الكبرى ويقع بالتحديد فى التى

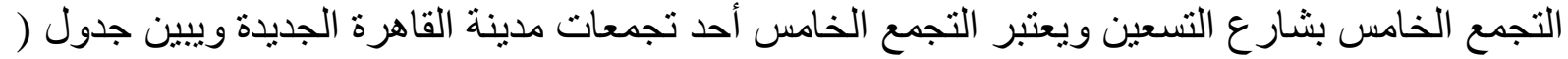

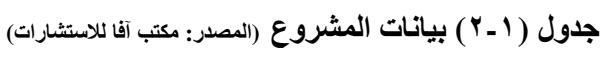

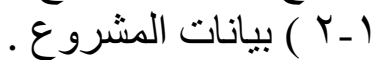

\begin{tabular}{|c|c|}
\hline \multicolumn{2}{|l|}{ 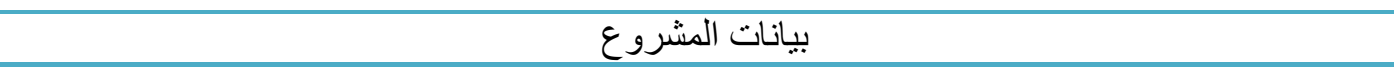 } \\
\hline 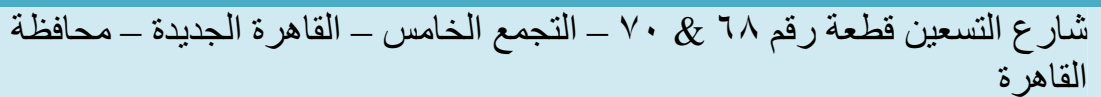 & 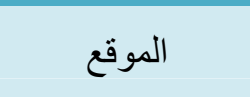 \\
\hline 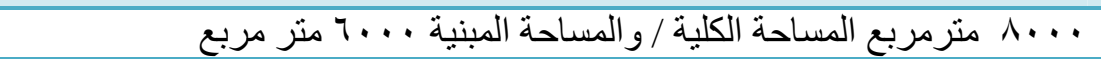 & المساحة \\
\hline مبنى إدارى & 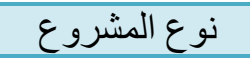 \\
\hline شركة عربية أوفيس بيلدينج & الماللك \\
\hline r بدروم + عطو ابق + سطح & مكونات المبنى \\
\hline مكتب آفا للاستشار ات & 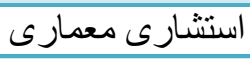 \\
\hline مكتب د/ مجدى كامل & استشارى MPE \\
\hline مكتب د / حاتم البحيرى & استشارى انشائى \\
\hline شركة اتريم للمقاو لات & المقاول التشطيبات \\
\hline شركة يونى تلك & مقاول MPE \\
\hline شركة عربية مكتب البناء & مقاول الإنشائى \\
\hline
\end{tabular}

ويوضح شكل ( ـ 1 ) خريطة الموقع (موقع مبنى التايكونز بشارع التسعين)

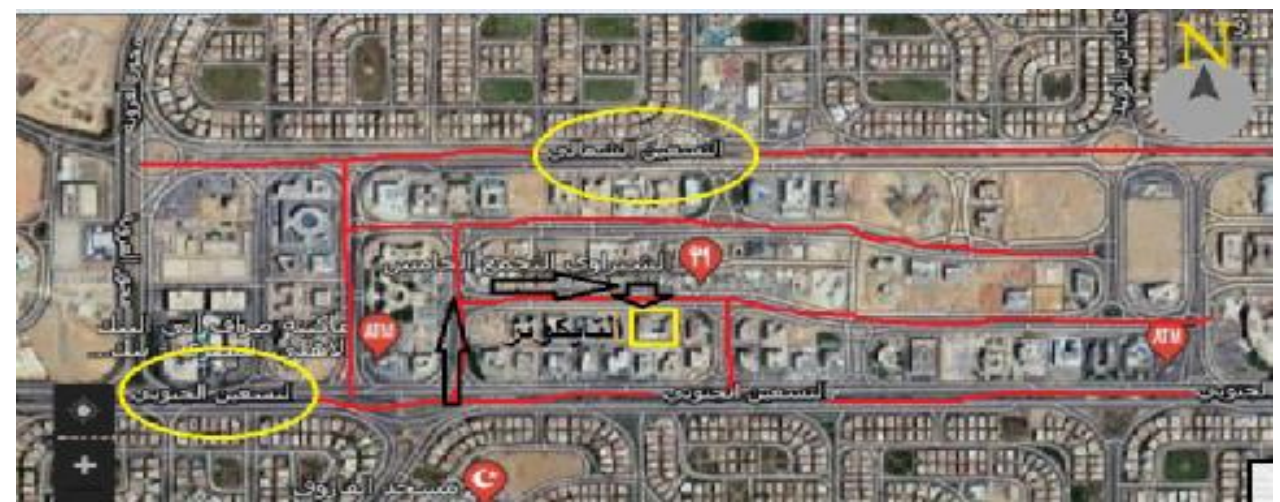

شكل (1 ـ ال) خريطة الموقع (موقع مبنى التايكونز بثارع التسعين)

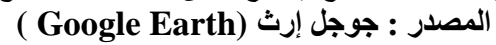




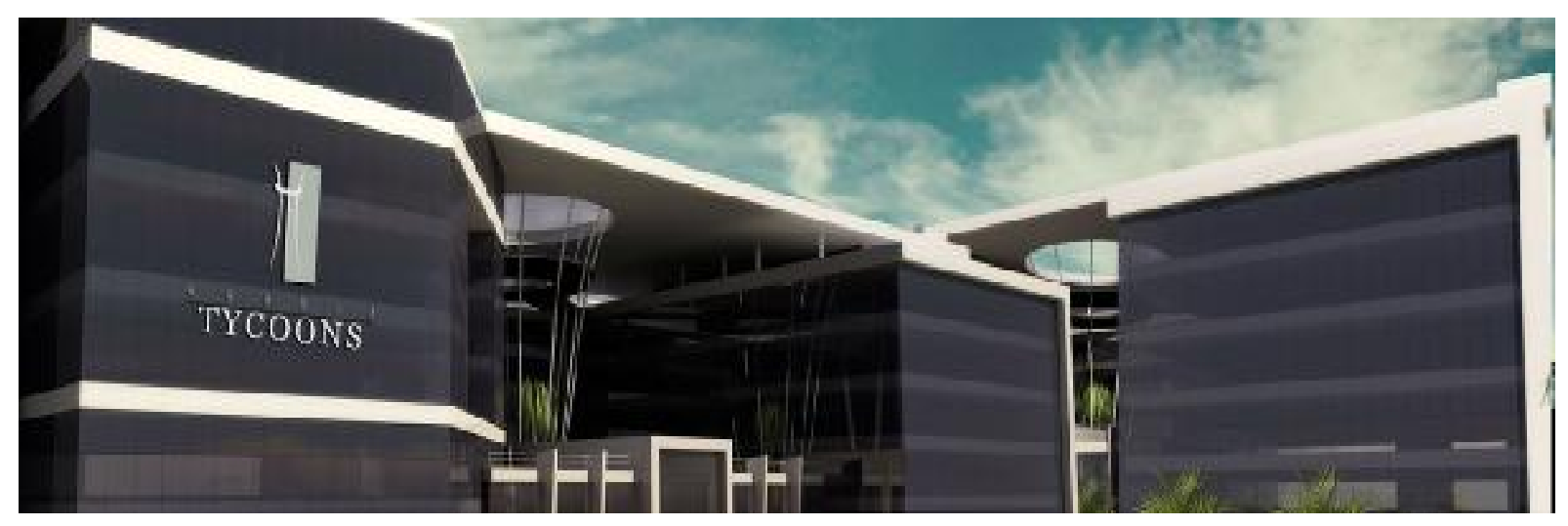

شكل (1 ب r ) واجهة أمامية لمبنى التايكونز

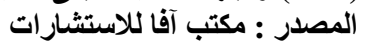

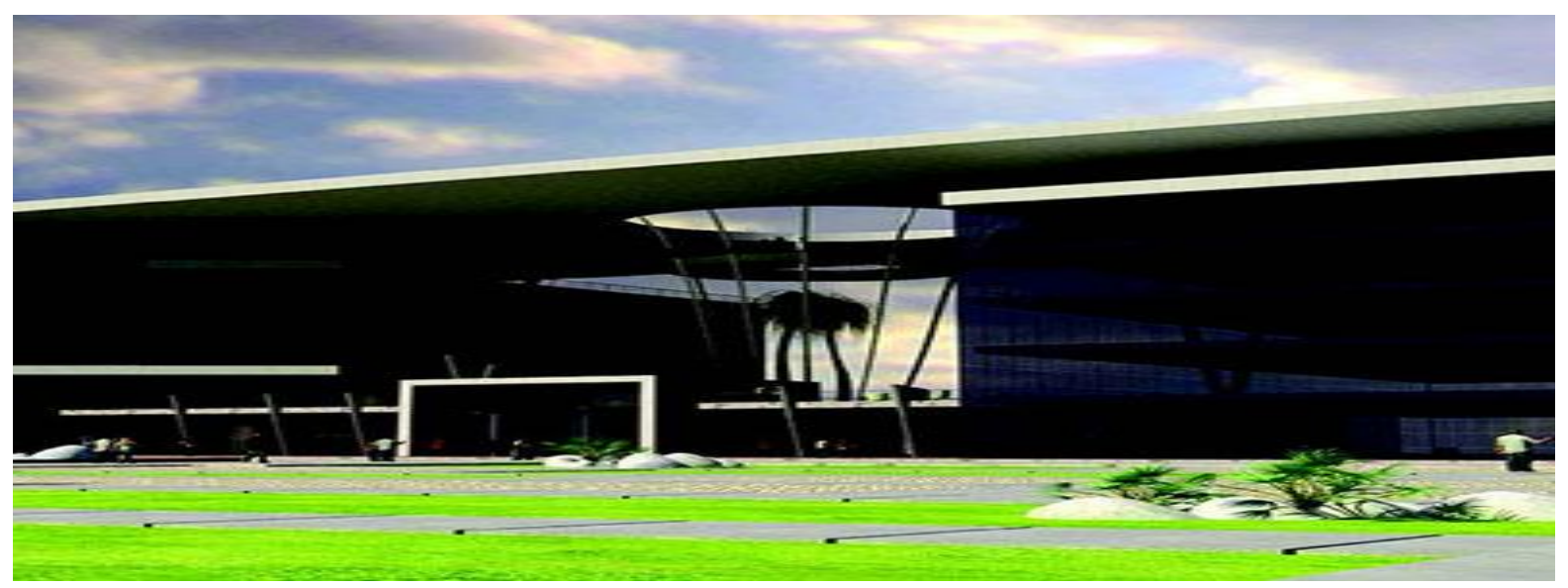

شكل ( ا-ب) واجهة أمامية لمبنى التايكونز

المصدر : مكتب آفا للاستشارات الاتينات

سوف يتناول البحث عرض المساقط الأفقية والوضع القائم للمبنى ويوضحه جدول ( إبـ) 


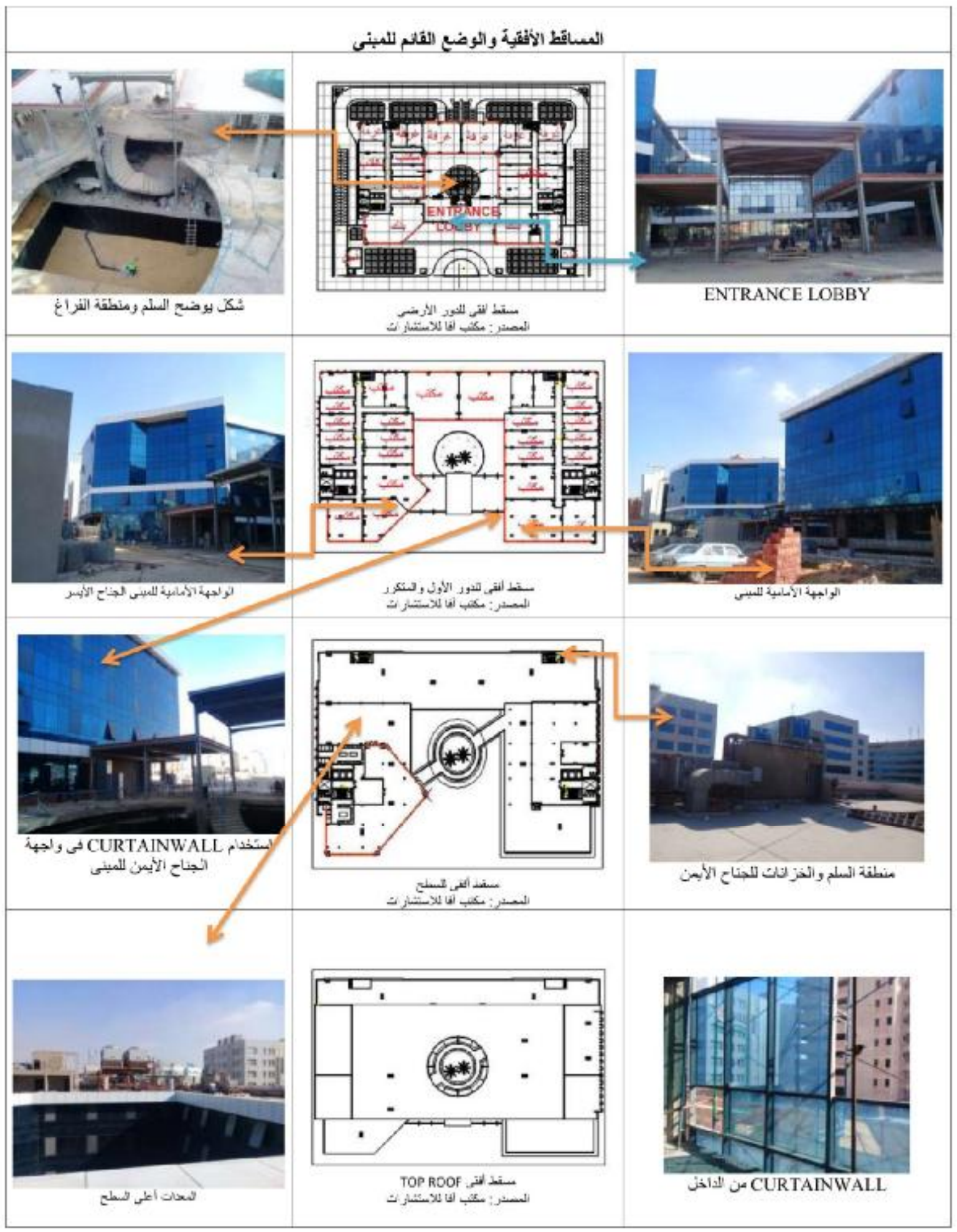

تم التقاط الصور بو اسطه الباحث ،بعد عرض البحث للوضع القائم سوف يتناول البحث قطاع للمبنى يوضح استخدام

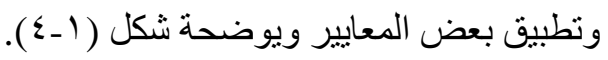




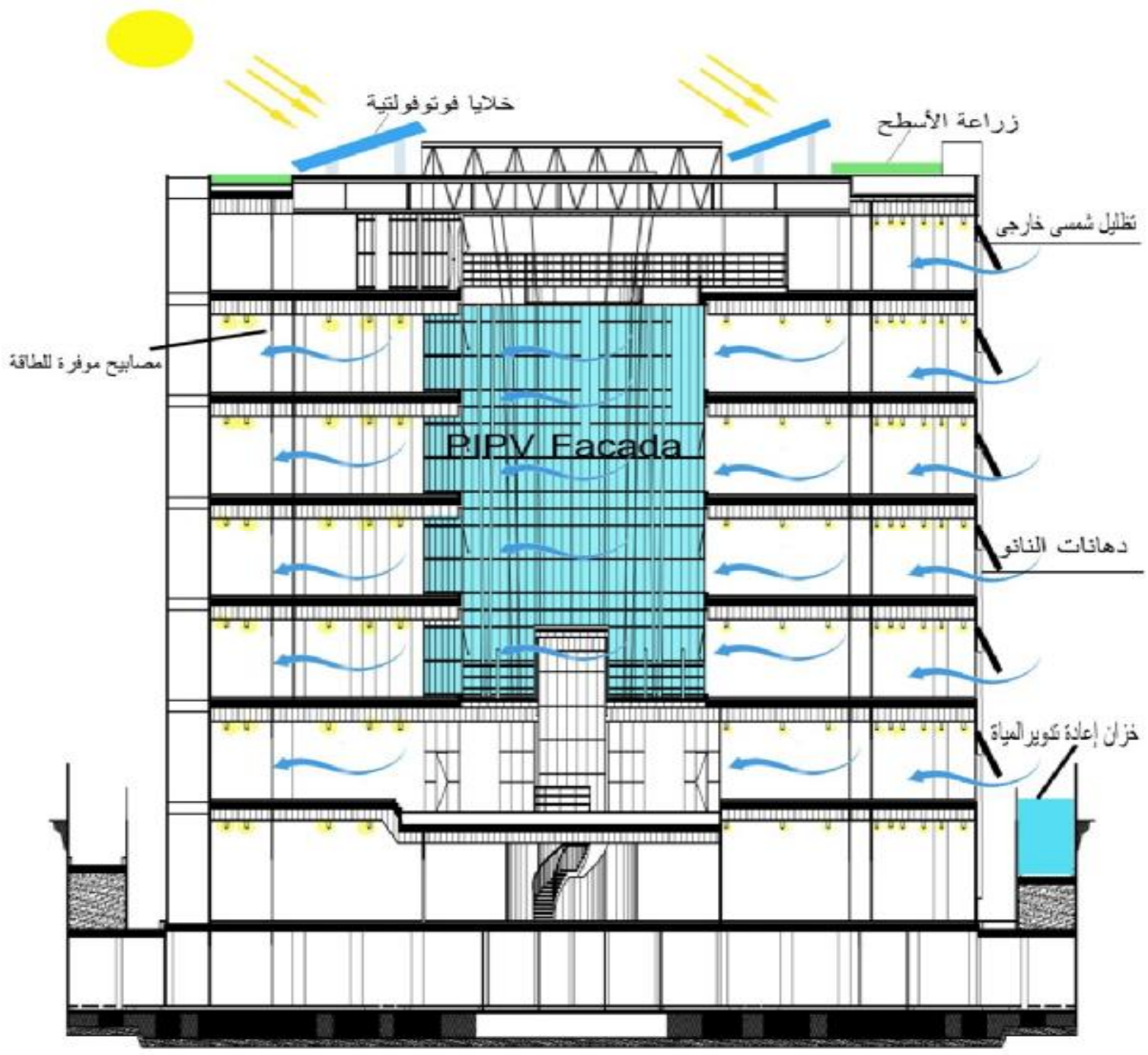

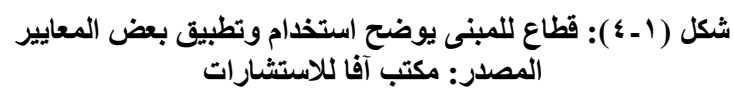

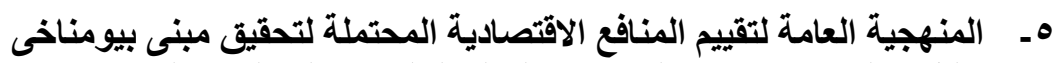

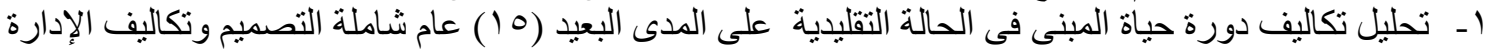
وتكاليف البناء وتكاليف الاستبدال و التشغيل و وتكاليف الصيانة الصيانة

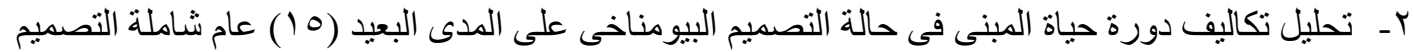

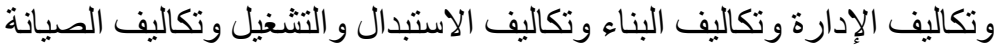
r- مقارنة تكاليف دورة حياة المبنى فى الحالة التقليدية وفى حالة استخدام التصميم البيومناخى لتحديد المنافع الاقتصنادية

7ـ - مقترح استخدام المعالجات البيومناخية المعمارية على مبنى التايكونز

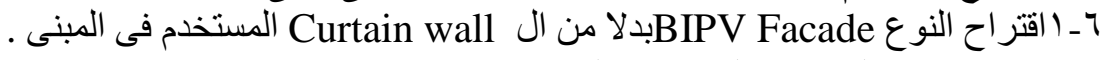

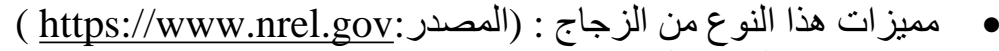

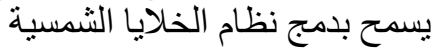
يتم تركيب الألو اح الكهروضوئية على النية الزجاج لتوليد الكهرباء من الطاقة الثنمسية

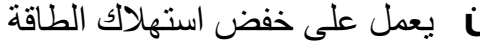


يعمل على استخدام الطاقة الثمسية كطاقة نظيفة بشكل فعال. لا حاجة للوقود، لا غاز العادم، لا انتاج الكربون ولا

يمكن توصيل الطاقة المولدة بالثبكة وتخفيف الضغط من إمدادات الطاقة.

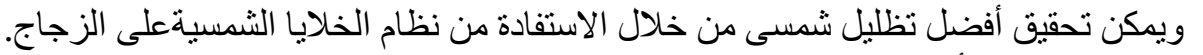

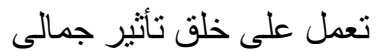

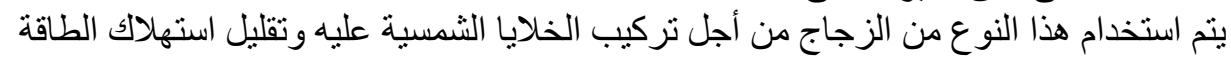

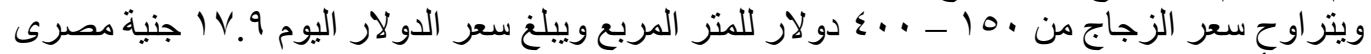

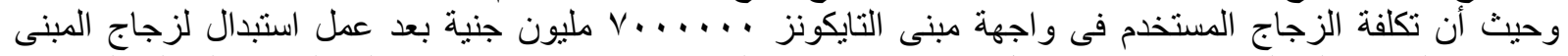

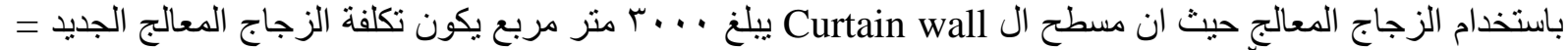
. 10.

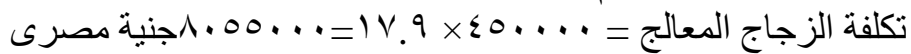

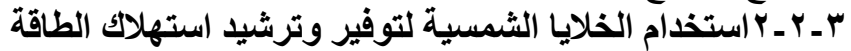

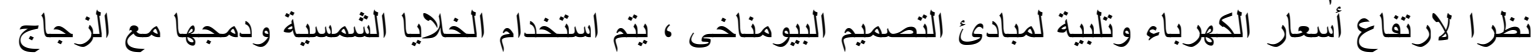

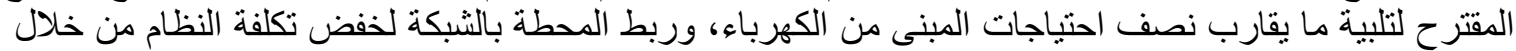

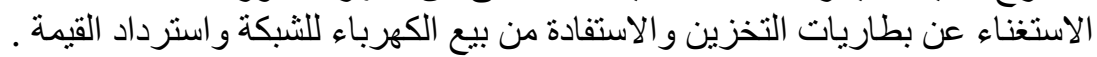

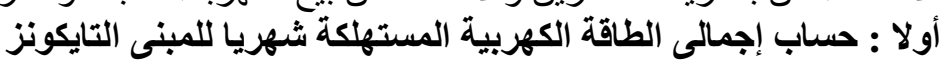

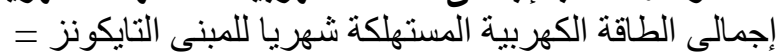

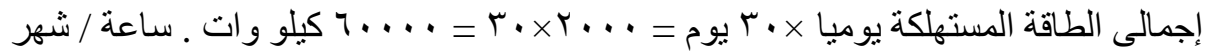

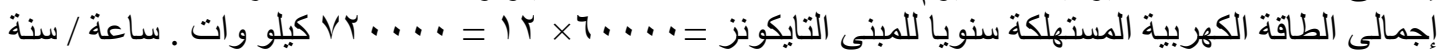

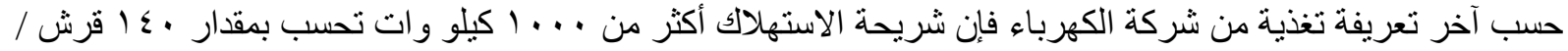

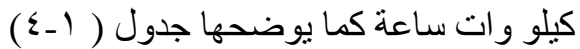

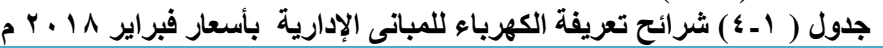

\begin{tabular}{|c|c|c|c|c|c|}
\hline أكثر من ... & $1 \ldots-7$. & $7 \ldots$ & ro... & $1 \ldots$ & شريحة الاستهلاك / شهر) \\
\hline $1 \leqslant$. & o & 97 & $\wedge \varepsilon$ & $\leqslant 0$ & 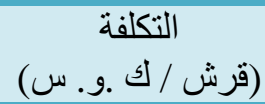 \\
\hline
\end{tabular}

تكلفة الأحمال الكهربية المستهلكة شهرياً للمبنى =

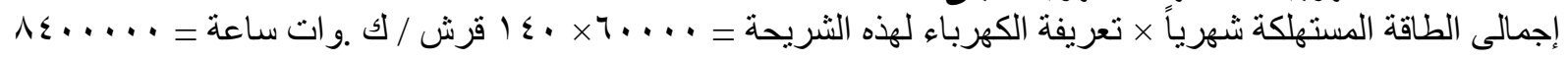

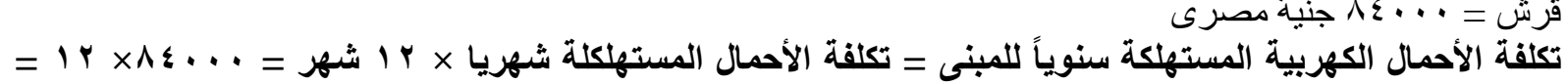

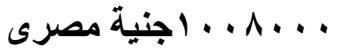

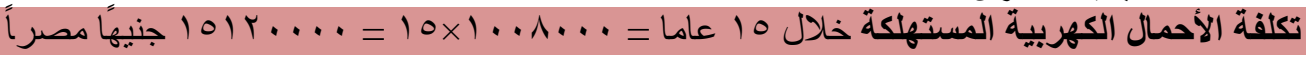

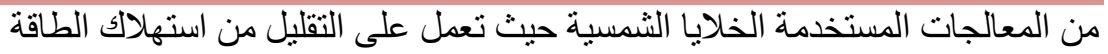

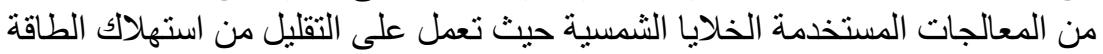

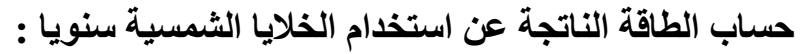

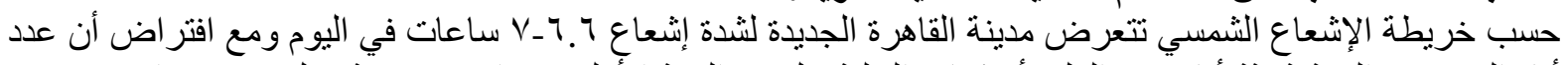

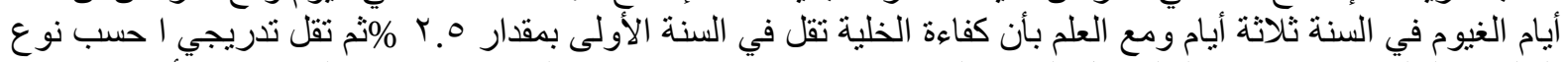

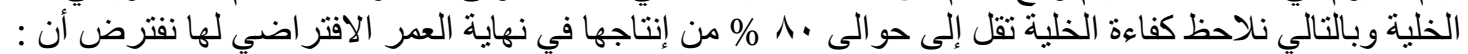

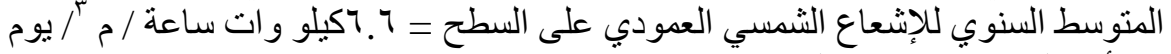

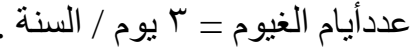
مقتر ح لاستخدام الخلايا الثمسية على الثئية مبنى التايكونز

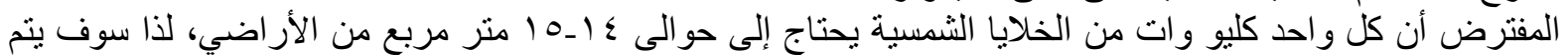

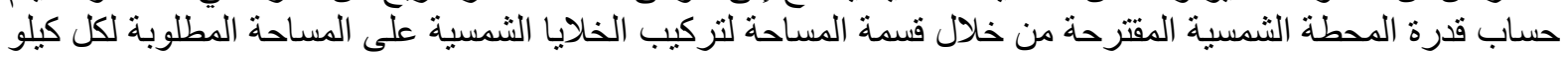

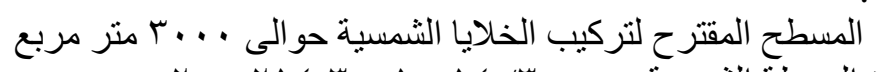

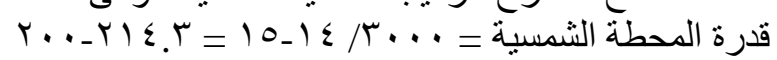

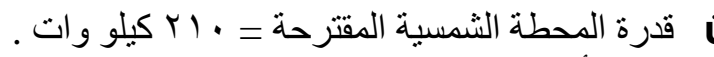

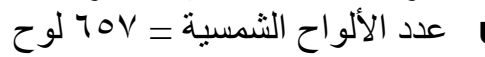

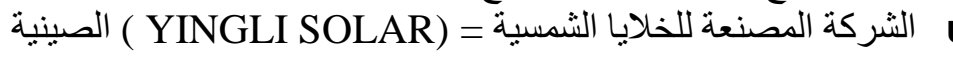




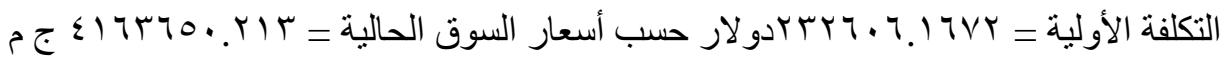

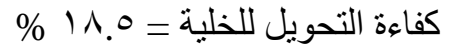

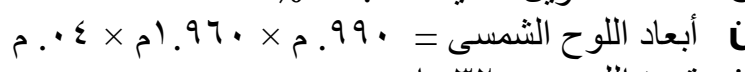

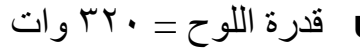

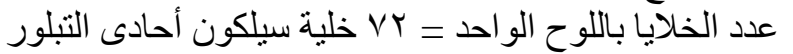

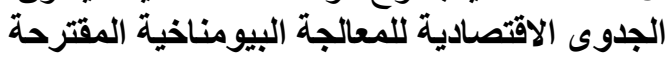

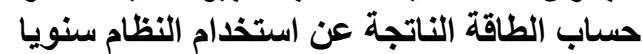

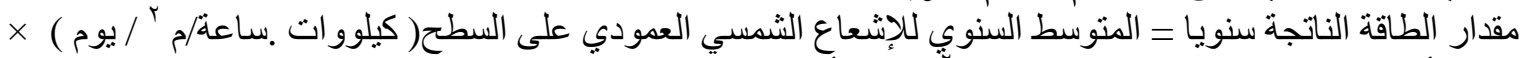

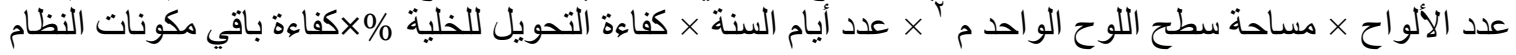

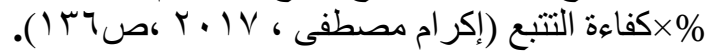

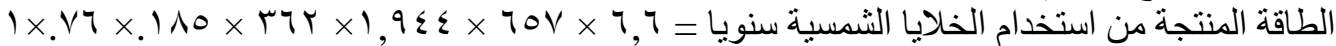

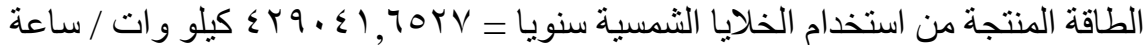

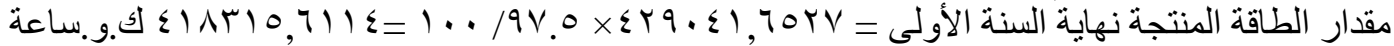

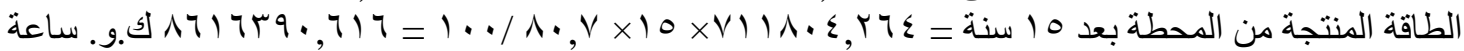

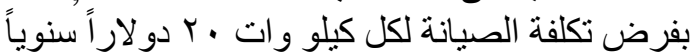

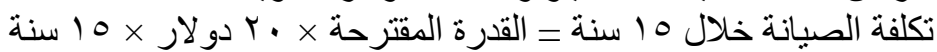

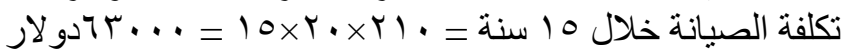

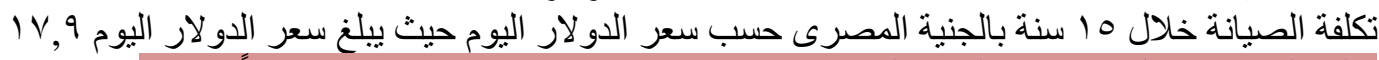

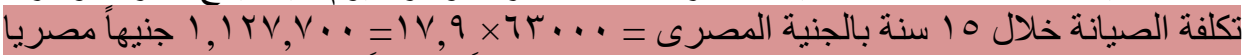

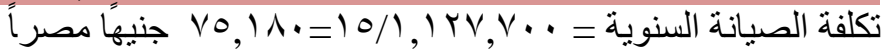

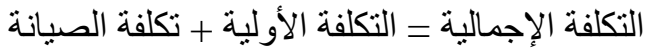

التكلفة الإجمالية =

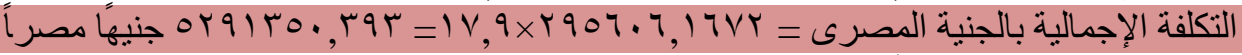

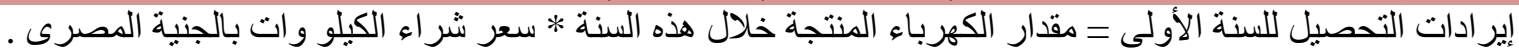

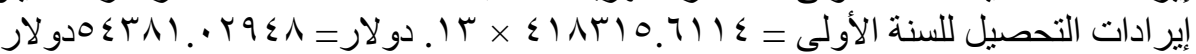

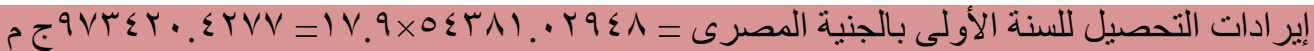

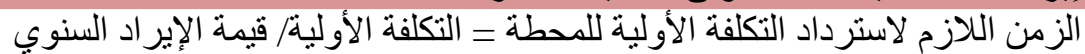

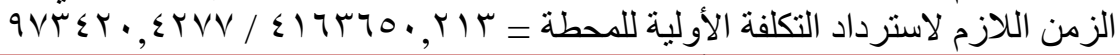

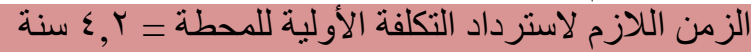

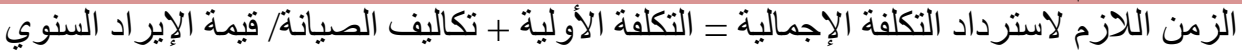

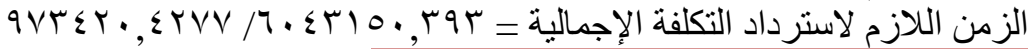

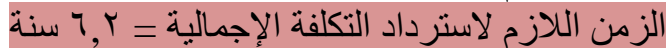

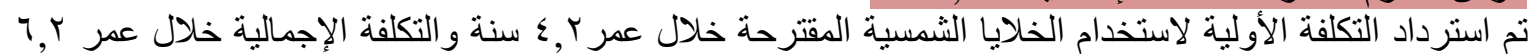

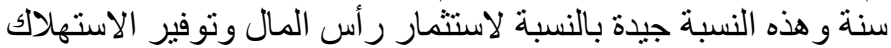

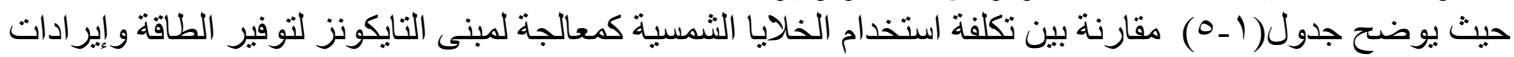
تحصيل الطاقة المنتجة من المحطة خلال 10 سنة بالجنية المصرى لتحقيق الكفاءة الاقتصادية لتئية 
جدول( ـ ـ ) مقارنة بين تكلفة استخدام الخلايا الثمسية كمعالجة لمبنى التايكونز لتوفير الطاقة وإيرادات تحصيل الطاقة المنتجة من المحطة (المصدر: الباحث) النمايكز

\begin{tabular}{|c|c|c|c|c|}
\hline \multicolumn{2}{|c|}{ إير ادات التحصيل } & \multicolumn{2}{|c|}{ تكلفة استخدام الخلايا الشمسية } & \multirow{2}{*}{ 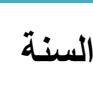 } \\
\hline ت تر اكمى & سنوى & ت تر اكمى & سنوى & \\
\hline$q V T \leqslant Y \cdot . \leqslant Y V V$ & $q V T \leq Y \cdot . \varepsilon r V V$ & EITrTO. YIT & हाтT & 1 \\
\hline $19 \leqslant 7 \wedge \leq \cdot .100$ & $q V T \leqslant Y \cdot . \Sigma T V V$ & 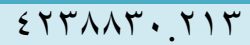 & Vo11. & r \\
\hline YqY. rTI. rAr & $q V T \leqslant Y \cdot . \leqslant Y V V$ & $\varepsilon \Psi) \varepsilon \cdot 1 \cdot . r \mid r$ & volı. & $r$ \\
\hline 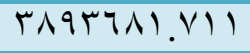 & $q V T \leqslant Y \cdot . \Sigma T V V$ & $\varepsilon r \wedge 919 \cdot . r \mid r$ & Vol1. & $\varepsilon$ \\
\hline$\varepsilon \wedge T \vee|\cdot Y|. T \wedge$ & $q \vee r \varepsilon r \cdot . \varepsilon r V V$ & $\varepsilon \varepsilon \tau \varepsilon r V \cdot . r \mid r$ & volA. & 0 \\
\hline O^E.OYY.0TT & $q V T \leqslant Y \cdot . \Sigma T V V$ & $\varepsilon 04900 ., r \mid r$ & volı. & 7 \\
\hline 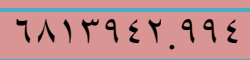 & $q \vee T \leqslant Y \cdot . \Sigma Y V V$ & $\varepsilon \pi) \leqslant V r \cdot . r \mid r$ & volA. & v \\
\hline VVAVYーT.EYT & $9 V T \leqslant Y \cdot . \Sigma T V V$ & $\varepsilon 7 \wedge 991 \cdot . r \mid r$ & volı. & $\Lambda$ \\
\hline$\wedge \vee \neg \cdot \vee \wedge r . \wedge \leq q$ & $q V T \leqslant Y \cdot . \leqslant T V V$ & $\varepsilon V 70 \cdot 9 \cdot . r \mid r$ & vol1. & 9 \\
\hline $9 V T \Sigma Y \cdot \varepsilon . Y Y V$ & $9 V T \leqslant Y \cdot . \Sigma T V V$ & $\varepsilon \wedge \varepsilon \cdot r \vee \cdot . r \mid r$ & volı. & 1. \\
\hline I.V・VTrะ.V & $Q V T \leqslant Y \cdot . \Sigma Y V V$ & $\leqslant q 10 \leqslant 0 ., r \mid r$ & volA. & 11 \\
\hline $11711 \cdot \leqslant 0.14$ & $q V T \leqslant Y \cdot . \leqslant T V V$ & $\varepsilon 99.7 r \cdot . r \mid r$ & volı. & ir \\
\hline $1 Y 70 \leqslant \leqslant 70.07$ & $Q V T \leqslant Y \cdot . \Sigma Y V V$ & $0.70 N 1 \cdot .1 T$ & volA. & ir \\
\hline 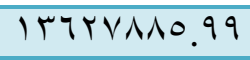 & $9 V T \leqslant Y \cdot . \Sigma T V V$ & $01 \leq .99 \cdot . Y 1 T$ & volı. & $1 \varepsilon$ \\
\hline $1 \leqslant 7.1 T \cdot \tau . \leqslant r$ & $q V T \leqslant Y \cdot . \leqslant T V V$ & OYITIV•.YIT & vol1. & 10 \\
\hline $100 V \leqslant V Y 7 . \wedge \leqslant$ & $9 V T \leqslant Y \cdot . \Sigma T V V$ & OYqITO. YIT & volı. & 17 \\
\hline $170 \leqslant \lambda I \leqslant V . Y V$ & $q \vee r \varepsilon r \cdot . \varepsilon r V V$ & OH & volA. & IV \\
\hline IVOYIOTV.V & $q V T \leqslant Y \cdot . \varepsilon T V V$ & $0 \leqslant \varepsilon|V| \cdot . r \mid T$ & Vol1. & 11 \\
\hline $1 \wedge \varepsilon q \leq q \wedge \wedge . \mid r$ & $q V T \leqslant Y \cdot . \Sigma Y V V$ & $0017 \wedge 9 \cdot . r 1 T$ & volA. & 19 \\
\hline $19 \leqslant 7 \wedge \leqslant .1 .00$ & $q V T \leqslant Y \cdot . \leqslant T V V$ & $009 T \cdot V \cdot . r / T$ & volı. & $r \cdot$ \\
\hline$r \cdot \Sigma \varepsilon \mid \wedge r \wedge . q \wedge$ & $q V T \leqslant Y \cdot . \leqslant T V V$ & OTTVYO. KIT & vol1. & YI \\
\hline Y) $\leqslant 10 r \leqslant 9 . \leqslant 1$ & $q V T \leqslant Y \cdot . \Sigma T V V$ & $O V \leqslant Y \leqslant T \cdot . r \mid r$ & volı. & rr \\
\hline 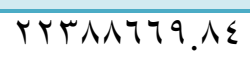 & $q V T \leqslant Y \cdot . \leqslant Y V V$ & ONIVTI. KIT & Vol1. & Yr \\
\hline YrTצr.q. TV & $q V T \leqslant Y \cdot . \leqslant T V V$ & O^ЯYVq. YIT & Volı. & $r \varepsilon$ \\
\hline$r \leqslant T r 001 \cdot .79$ & $q V T \leqslant Y \cdot . \Sigma T V V$ & 097VqV. KIT & Vol1. & ro \\
\hline
\end{tabular}

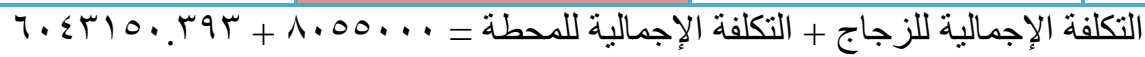

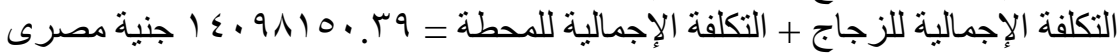

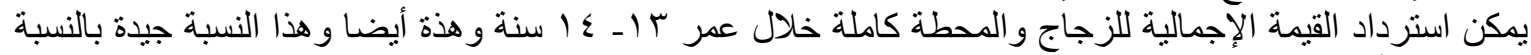

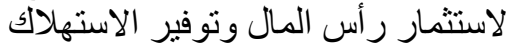

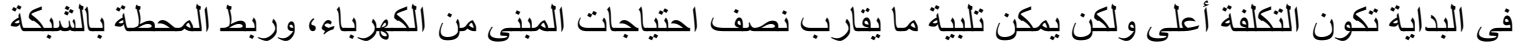

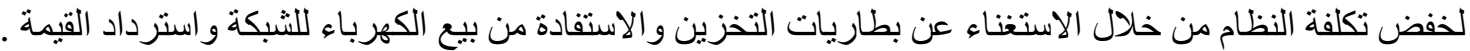

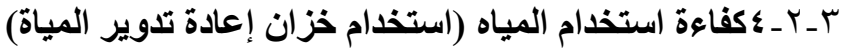

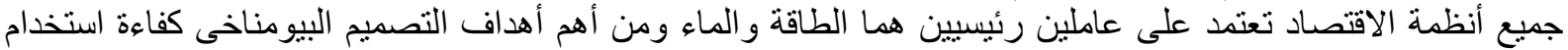

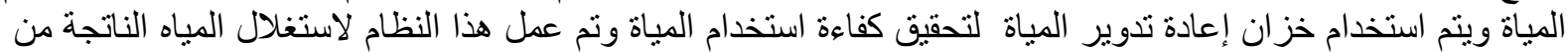

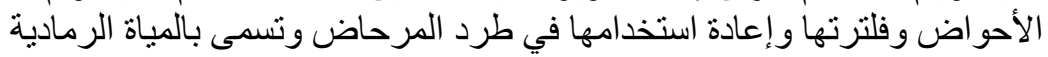

حساب استهلاك مبنى التايكونز للمياه

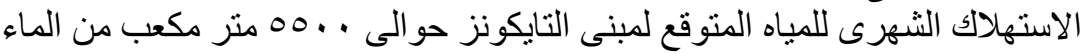

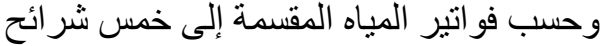

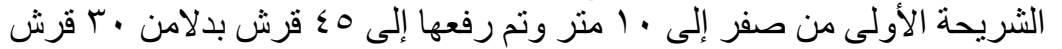

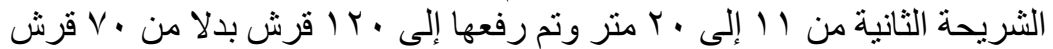

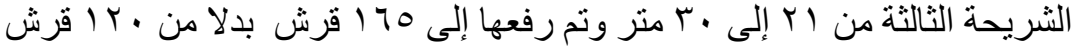

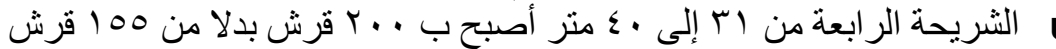

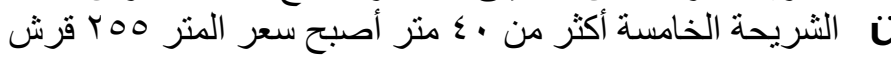




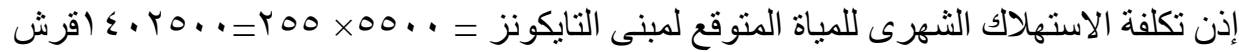

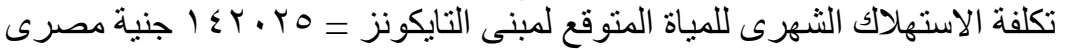

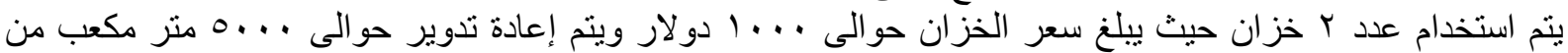
الماء

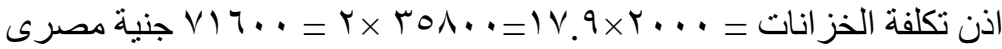

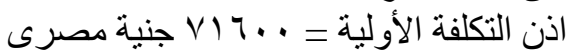

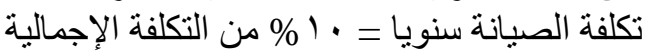

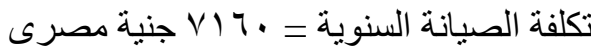

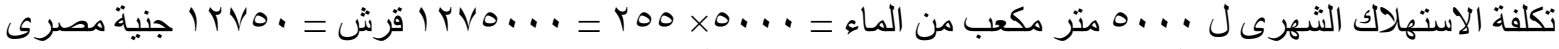

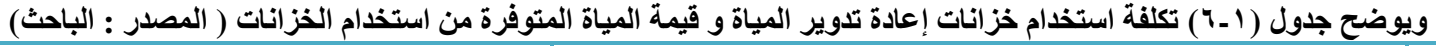

\begin{tabular}{|c|c|c|c|c|}
\hline \multicolumn{2}{|c|}{ قيمة المياة المتوفرة من استخدام الخزانات } & \multicolumn{2}{|c|}{ تكلفة استخدام خزانات إعادة تدوير المياة } & \multirow{2}{*}{ السنة } \\
\hline تر اكمى & سنوى & تر اكمى & سنوى & \\
\hline I YVO. & I TVO. & V) .. & $\vee 17 \ldots$ & $r$ \\
\hline Y00.. & I YVO. & VAVT. & V17. & $r$ \\
\hline rAYO. & I TVO. & 109r. & VIT. & $\varepsilon$ \\
\hline $01 \ldots$ & I YVO. & $9 r \cdot \Lambda$. & V). & 0 \\
\hline TrVo. & I YVO. & $1 \ldots r \leqslant$. & VIT. & 7 \\
\hline V70.. & I rVO. & $1 \cdot v \leqslant \ldots$ & V17. & $\mathrm{v}$ \\
\hline AqYo. & I YVO. & $11 \leqslant 07$. & VIT. & $\Lambda$ \\
\hline I. r... & I YVO. & IYIVY. & VIT. & 9 \\
\hline $11 \leq \vee 0$. & I YVO. & 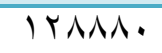 & V). & 1. \\
\hline I YVO.. & I YVO. & $1 \% \longleftarrow \cdot \varepsilon$. & VIT. & 11 \\
\hline $1 \leqslant .10$. & I TVO. & $1 \leqslant T Y \ldots$ & VIT. & Ir \\
\hline $104 \ldots$ & I YVO. & $10 . \mathrm{Mr}$. & V). & ir \\
\hline $170 \vee 0$. & I YVO. & lOVOY. & VIT. & $1 \varepsilon$ \\
\hline IVAo.. & I YVO. & $17 \leq 7 \wedge$. & VIT. & 10 \\
\hline $191 \% 0$. & I YVO. & IVIAE. & VIT. & 17 \\
\hline$r \cdot \varepsilon \ldots$ & I YVO. & $1 \vee 9 \ldots$ & VIT. & IV \\
\hline YITVO. & I YVO. & $1 \wedge 717$. & VIT. & 11 \\
\hline Yrq0.. & I YVO. & . & V17. & 19 \\
\hline TEYTO. & I YVO. & $r \ldots \leqslant \Lambda$. & VIT. & $r$. \\
\hline roo... & I YVO. & r.VIE. & VIT. & YI \\
\hline YTVVO. & I YVO. & $r l \leq \Lambda \ldots$ & VIT. & Yr \\
\hline$r \wedge .0 \ldots$ & I YVO. & YYI97. & VIT. & rr \\
\hline rqrro. & I YVO. & TY197. & VIT. & $r \xi$ \\
\hline$r .7 . .$. & I YVO. & rrqIr. & VIT. & ro \\
\hline
\end{tabular}

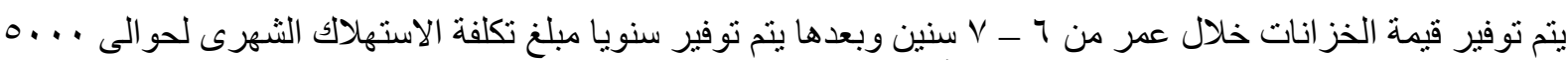

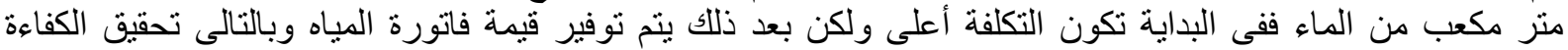
الاقتصادية من تحقيق كفاءة استخدام المباه

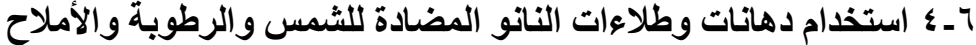

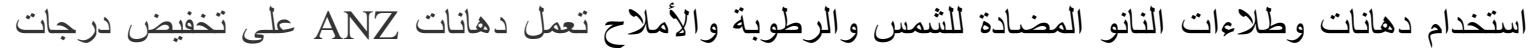

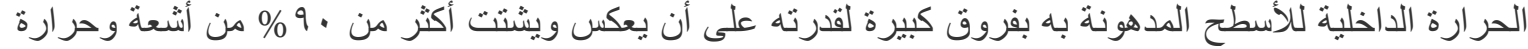

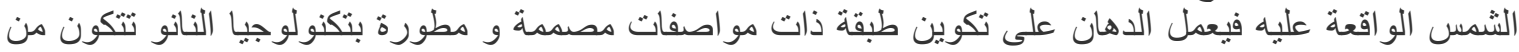

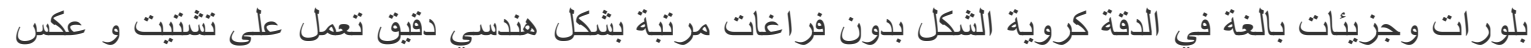
أثنعة الثمس وحر ارتها عن السطح المدهون ، وبالتالي تنخفض درجة الحرارة الداخلية للسطح المدهون عن الدئة 
الطبيعية درجة مئوية وهو عبارة عن دهان شفاف لزجاج المبانى كما يوفر في استهلاك الوقود المستخدم في التبريد أو

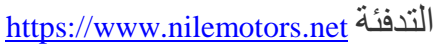

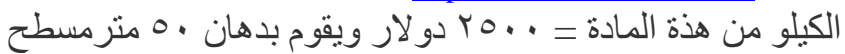

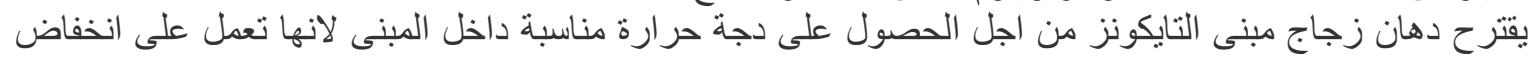

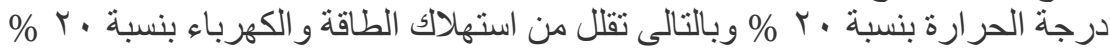

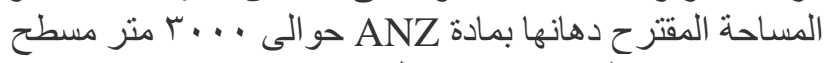

ANZ بل...

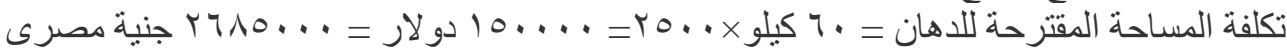

ويوضح جدول ( V-1) تكلفة المساحة المقترحة للاهان و القيمة المتوفرة فى استهلاك الطاقة (المصدر: الباحث)

\begin{tabular}{|c|c|c|c|}
\hline \multicolumn{2}{|c|}{ القيمة المتوفرة في استهلاك الطاقة } & تكلفة المساحة المقترحة للاهان & \multirow{2}{*}{ 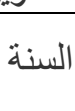 } \\
\hline تر اكمى & 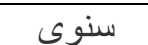 & تر اكمى تر تر ت & \\
\hline$r .17 \ldots$ & $r .17 \ldots$ & r $410 \ldots$ & 1 \\
\hline E.TY.. & $r .17 \ldots$ & r $710 \ldots$ & r \\
\hline $7 \cdot \leq \wedge \ldots$ & $r .17 \ldots$ & rฯ10... & 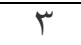 \\
\hline$\Lambda \cdot 7 \varepsilon \cdots$ & $r \cdot 17 \ldots$ & rTAO... & $\varepsilon$ \\
\hline $1 \ldots 1 \ldots$ & $r .17 \ldots$ & r\10... & 0 \\
\hline $14.97 \ldots$ & $r .17 \ldots$ & Y $710 \ldots$ & 7 \\
\hline $1 \leqslant \|, \ldots$ & $r .17 \ldots$ & r\10... & V \\
\hline $171 Y \wedge \ldots$ & $r .17 \ldots$ & r $710 \ldots$ & $\Lambda$ \\
\hline$|\wedge| \leq \varepsilon \ldots$ & $r .17 \ldots$ & rT10... & 9 \\
\hline$\overline{Y .17 \ldots}$ & $r .17 \ldots$ & Y $710 \ldots$ & 1. \\
\hline YYIVT.. & $r .17 \ldots$ & Y $410 \ldots$ & 11 \\
\hline$r \leqslant 19 r \ldots$ & $r .17 \ldots$ & Y $410 \ldots$ & IT \\
\hline rqr.A.. & $r .17 \ldots$ & r $710 \ldots$ & $\pi$ \\
\hline YAYYE.. & $r .17 \ldots$ & Y $710 \ldots$ & $1 \leqslant$ \\
\hline$r \cdot r \varepsilon \ldots$ & $r .17 \ldots$ & rฯA0... & 10 \\
\hline TrYOT.. & $r \cdot 17 \ldots$ & $r 710 \ldots$ & 17 \\
\hline$r \leqslant Y V T \ldots$ & $r .17 \ldots$ & $r 710 \ldots$ & IV \\
\hline rฯrAА.. & $r .17 \ldots$ & rฯ10... & 11 \\
\hline 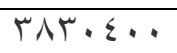 & $r .17 \ldots$ & 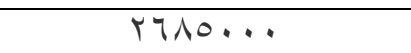 & 19 \\
\hline$\varepsilon \cdot r\} \ldots$ & $r .17 \ldots$ & r $410 \ldots$ & r. \\
\hline ะイルน .. & $r .17 \ldots$ & $r 710 \ldots$ & Y) \\
\hline$\varepsilon \leqslant$ TOY.. & $r .17 \ldots$ & Yฯ10... & Tr \\
\hline ะフルฯ^.. & $r .17 \ldots$ & rฯ^०... & Tt \\
\hline 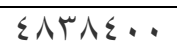 & $r .17 \ldots$ & rฯ^०... & $r \xi$ \\
\hline $0 . \varepsilon \ldots$ & $r .17 \ldots$ & Y Y10... & TO \\
\hline
\end{tabular}

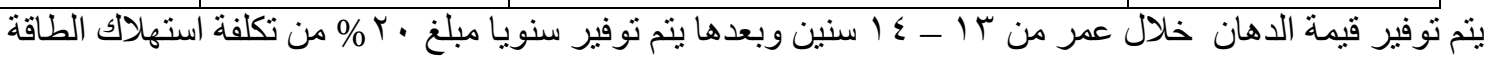

\section{ا ــ زراعة سطح المبنى ومدخل المبنى}

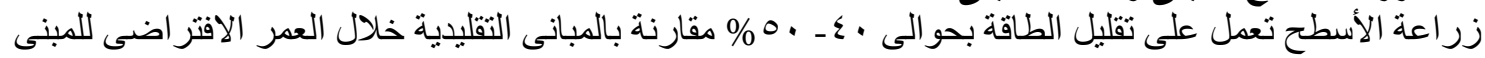
http://Www.alkhaleej.ae

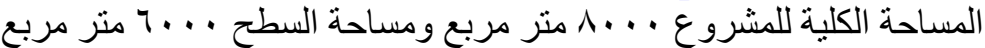
بفرض زر اعة . . . . متر مربع من السطح وزر اعة . . .0 متر مربع من المدخل الخاص بالمبنى حيث أن تكلفة

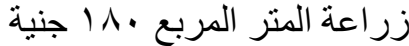

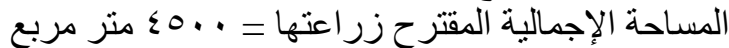

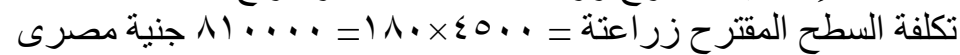

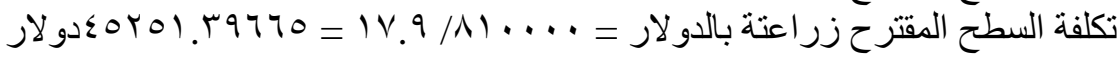


نفرض أن تكلفة الصيانة سنويا ـ أ (\% من التكلفة الإجمالية

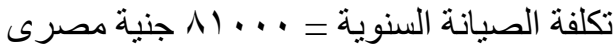

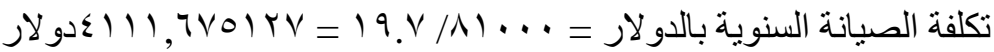

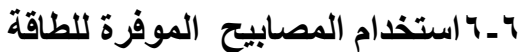

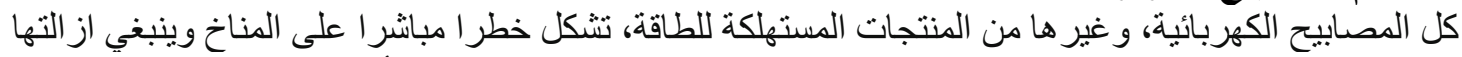

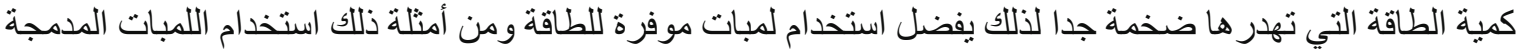

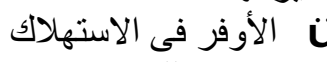

ومن مميز اتها

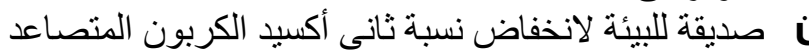

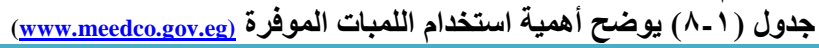

\begin{tabular}{|c|c|c|c|}
\hline قدرة اللمبة العادية & قدرة اللمبة المدمجة & 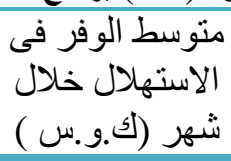 & 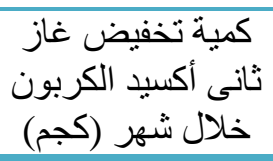 \\
\hline r. & $1 \cdot-1$ & $V, \varepsilon \varepsilon$ & 0 \\
\hline 7. & $10_{-11}$ & $11, Y \wedge$ & $V, 0$ \\
\hline Vo & $r \cdot-11$ & $1 T, \leqslant \varepsilon$ & 9 \\
\hline $1 \ldots$ & YO_K. & 11,7 & $1 T, \varepsilon$ \\
\hline
\end{tabular}

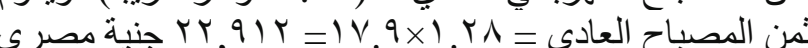

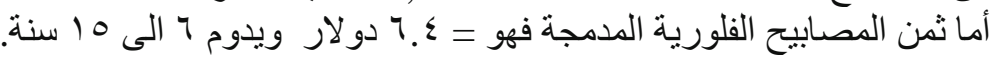

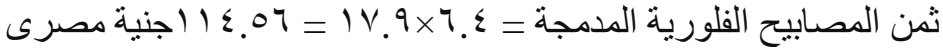

/gwwwreenpeace.org/arabic/campaigns/climate-change/energy_efficiency/lightbulbs-q-and-a.)

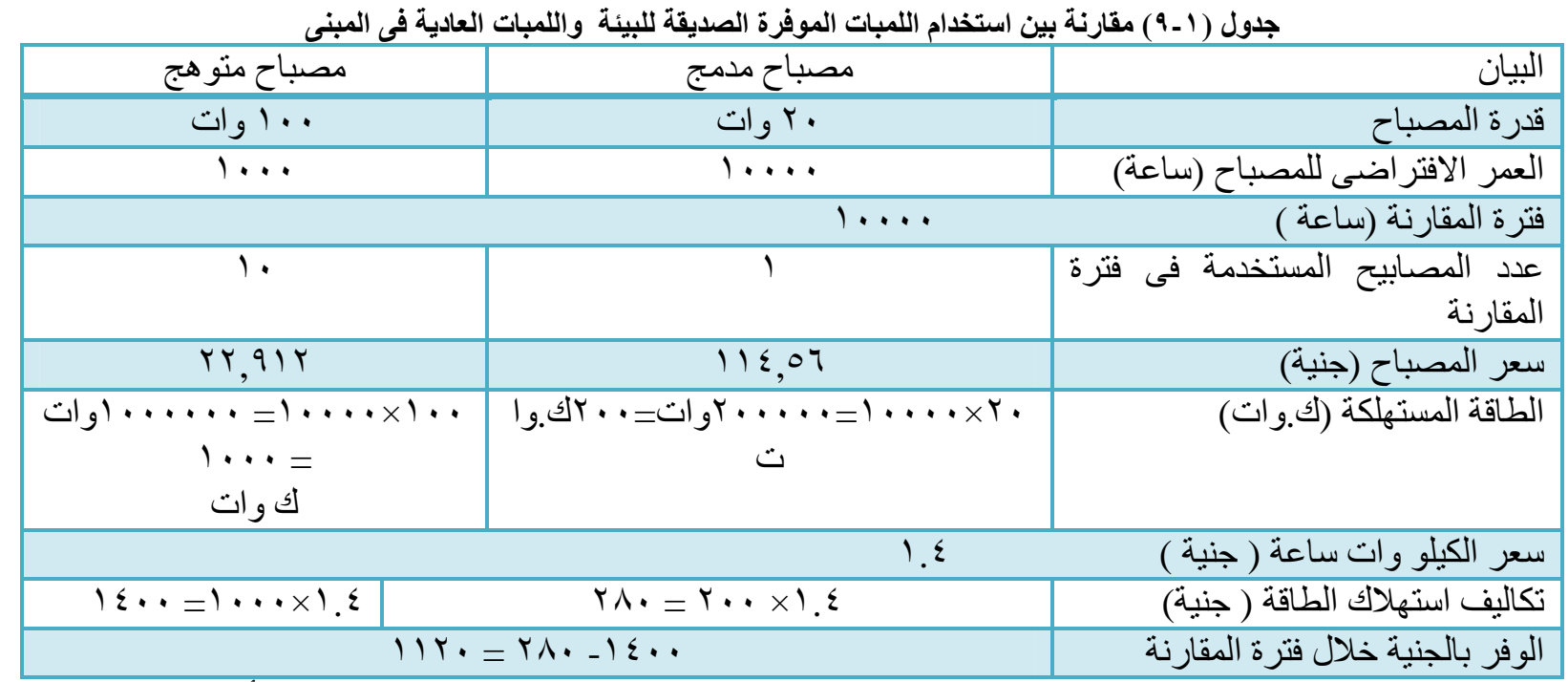

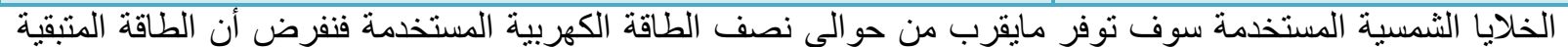

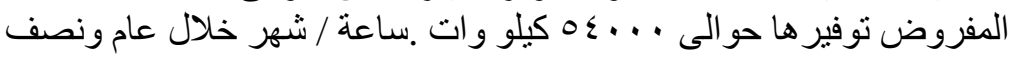

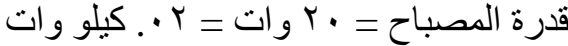

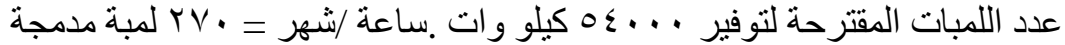

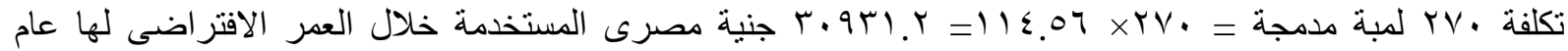

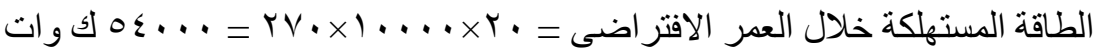

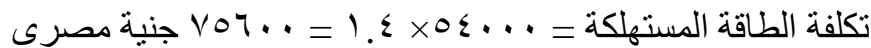

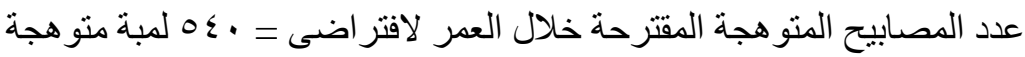

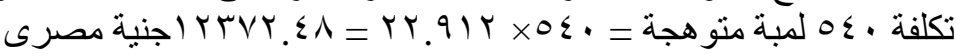




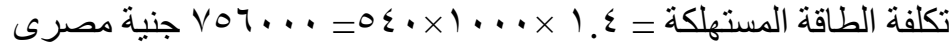

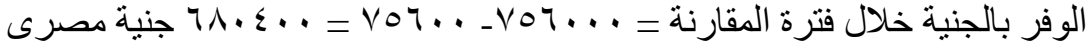

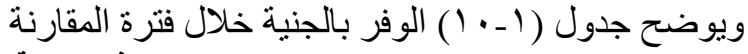
جدول ( ا ـ - 1) الوفر بالجنية خلال فترة المقارنة (المصدر : الباحث)

\begin{tabular}{|c|c|c|}
\hline مصباح منو هج & مصباح مدمج & 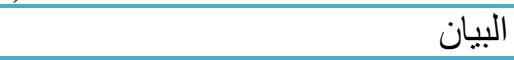 \\
\hline . . أوات & ت بوات & قدرة المصباح \\
\hline $1 \ldots$ & $1 \ldots$ & العمر الافتر اضى للمصباح (ساعة) \\
\hline \multicolumn{3}{|r|}{ فترة المقارنة (ساعة ) } \\
\hline $0 \leqslant$. & TV. & الدقارنة المصابيح المستخدمة فى فترة \\
\hline rr,qाr & $11 \leqslant, 07$ & سعر المصباح (جنية) \\
\hline \multicolumn{3}{|r|}{ سعر الكيلو وات ساعة ( جنية ) } \\
\hline vor... & vor.. & تكاليف استهلاك الطاقة ( جنية) \\
\hline \multicolumn{2}{|c|}{. . . . . جنية مصرى } & الوفر بالجنبة خلال فترة المقارنة \\
\hline
\end{tabular}

جدول (1 ـ 1 1) حساب تكاليف المعالجات وتحديد قيمة الزيادة (المصدر : الباحث)

\begin{tabular}{|c|c|c|c|}
\hline تكلفة الدهان & تكلفة الخز انات & تكلفة الزجاج المعالج & تكلفة الخلايا الثمسية \\
\hline r $710 \ldots$ & $\vee \backslash 7 \ldots$ & $1.00 \ldots$ & שוT \\
\hline ن نسبة الزيادة & 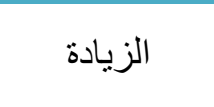 & تكلفة استخدام المصدابيح & تكلفة زر اعة السطح \\
\hline$\%$ & $r q, V \cdot 1,1 r^{\prime}$ & r.9r।ᄉ & $191 \ldots$ \\
\hline & & צr 1, • • 1 1 جنيهاً مص & \\
\hline
\end{tabular}

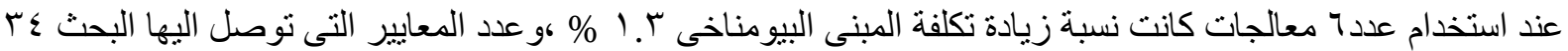
بفرض أن الأوزان النسبية متساوية و تم عمل تطبيق عملى على 7 معالجات وكانت الزيادة ب, ا اذا الزيادة النسبية للمعايير $v, r \wedge=1 . r \times 0,7=r \varepsilon$

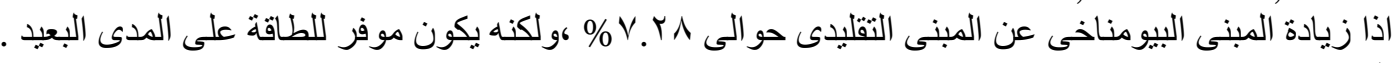
أوضحت الدر اسة التطبيقية التى أجريت على مشروع مبنى التايكونز العديد من المعايير التى تعمل على تطوير استخدام التصميم البيومناخى بطريقة اقتصادية لتحقيق استدامة المبنى والحصول على أكبر قدر من الراحة الحرارية ويمكن

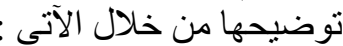
در اعاة اعتبار ات الموقع من حيث مر اعاة الظروف المناخية و التوجية الجيد ، واستخدام النظم الثمسية السالبة

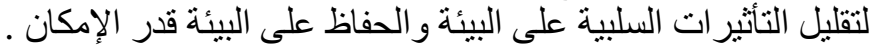

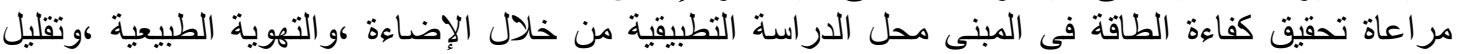
الحمل الحرارى على المبنى ،واستخدام مواد البناء التى تتميز بالعزل الحرارى العالى فى تكوين الغلاف

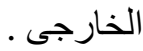
استخدام مو اد البناء الطبيعية ساعد فى تقليل الدهانات وأعمال التشطيب الخارجى مما ساعد فى تحسين جودة البيئة الداخلية والوصول البئل إلى إلى مبنى بيئى صحى ،و التقليل من التكلفة الاقتصادية، وتحقيق التكامل مع البيئة و الحد من التأثبر السلبى على البيئة .

\section{التو صباث}

د تحديد وجهات النظر و التحليلات التى تساعد على سد الفجوة بين المعماريين و الاقتصادبين وأصحاب المصلحة 


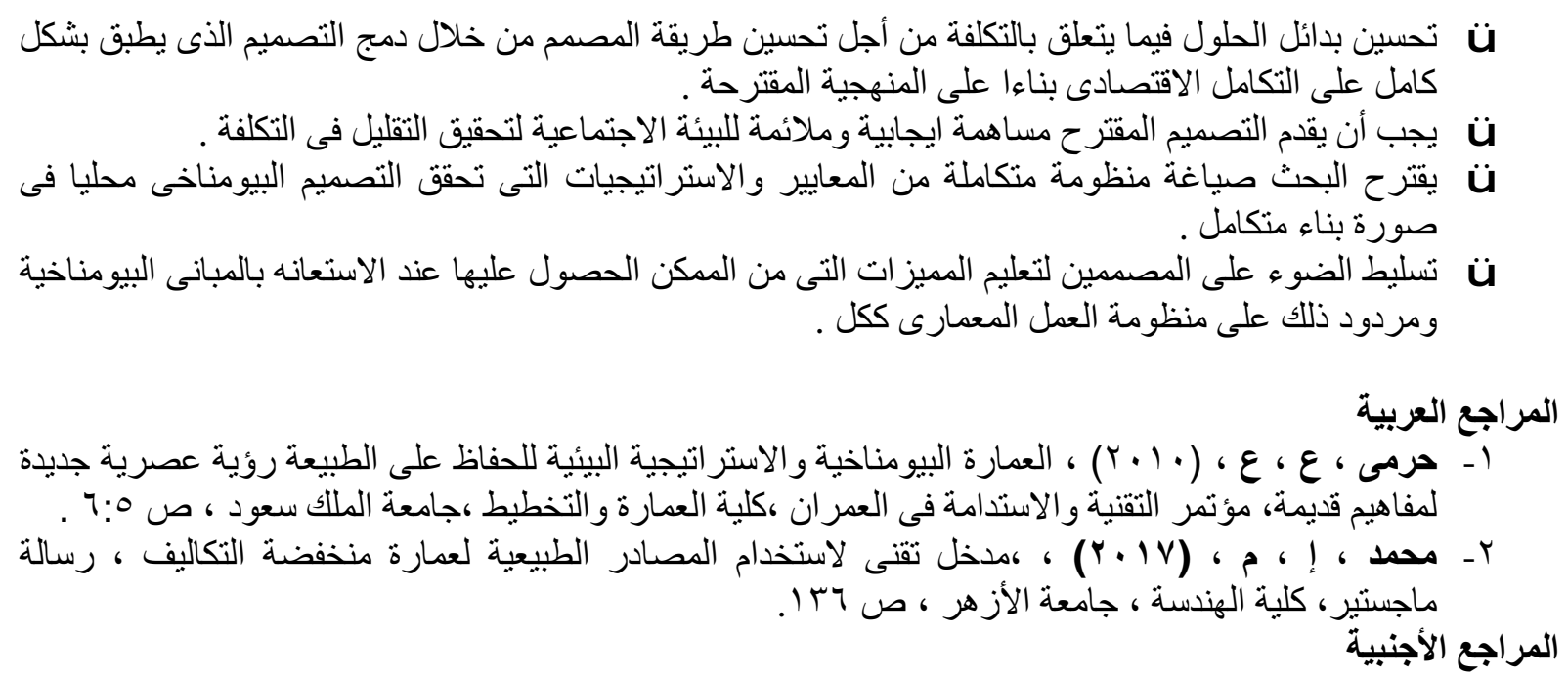

3- Hyde,R,H.( 2008) . Bioclimatic Housing. in the uk and USA, Earthscan.

1. Baby, R, B,(2018), Retrieved from https://www.nilemotors.net https://www.nrel.gov Retrieved from . Gaiddon, B, H, (2017)

2. Hansen, G,H (April 25, 2018) | Agriculture, Environment, Green Building, Sustainable DevelopmentRetrieve from dhttp://www.alkhaleej.ae .

3. Mitchell ,c,M,(2018), Retrieved from www.meedco.gov.eg. Mitchell ,c,M,(2018), Retrieved from www. climate.org. 